\title{
1 CORRECTING FOR SYSTEMATIC BIASES IN GCM SIMULATIONS IN THE
}

2 FREQUENCY DOMAIN

3 Ha Nguyen ${ }^{\mathrm{a}}$, Rajeshwar Mehrotra ${ }^{\mathrm{a}}$ and Ashish Sharma ${ }^{\mathrm{a}, *}$

$4{ }^{a}$ Water Research Centre, School of Civil and Environmental Engineering, The University

5 of New South Wales, Sydney NSW 2052, Australia.

6 *Corresponding author: Ashish Sharma, Water Research Centre, School of Civil and

7 Environmental Engineering, The University of New South Wales, Sydney NSW 2052,

$8 \quad$ Australia. (A.Sharma@unsw.edu.au)

10 Abstract

11 Bias correction is considered as a critical post-processing step to remove systematic errors and improve the quality of General Circulation Model (GCM) simulations before their use in climate change impact assessment applications. A majority of the bias correction approaches correct for biases either at a single time scale or at multiple prespecified time scales. An inappropriate or insufficient selection of time scales may lead to improper or sub-optimal bias corrected outputs, especially when persistence attributes across a range of scales are of interest. In this paper, we present a new bias correction approach that works in the frequency space and is independent of specific time scales. The approach is named as frequency-based bias correction (FBC). The usefulness of the approach is demonstrated by applying it to the monthly rainfall simulations of MIROC5

21 GCM over Australia and comparing the results with two other approaches, namely, empirical quantile mapping and recursive nesting bias correction, in cross validation. The comparison is based on the reproduction of various observed distribution and persistence 
performance in terms of reproducing the first- and second- order moments of observed precipitation time series, however, outperforms with regard to persistence attributes. The approach shows high potential for use in downscaling and other climate change impact assessment studies, especially for the planning and design of hydrologic systems that are sensitive to the characterisation of persistence in the hydrologic time series.

Key words: General circulation models; downscaling; biases in atmospheric variables; recursive nesting bias correction; empirical quantile mapping; frequency-based bias correction.

\section{Introduction}

Quantifying climate change impacts on water resources systems at regional or catchment scales is very important in water resources planning and management of hydrological extremes (floods and droughts) (Chen et al., 2013; Haerter et al., 2011; Johnson and Sharma, 2011; Johnson and Sharma, 2012; Mehrotra and Sharma, 2010; Mehrotra and Sharma, 2012). General circulation models (GCMs) are regarded as the primary tool available to achieve the above purpose (Chen et al., 2013; Johnson and Sharma, 2009; Maraun et al., 2010). However, there are a number of key limitations that restrict the direct use of GCM outputs. The first important limitation relates to the spatial scale mismatch between the coarse resolutions of GCMs and the finer scale requirement of the hydrological models (Wood et al., 2004). Spatial downscaling techniques are available to bridge this gap and transfer the coarser scale GCM outputs to the finer scale (Fowler et al., 2007). The second core limitation relates to the presence of systematic biases in the GCM outputs. Despite much advancement in our understanding of the science and computing resources, various assumptions and limitations in processes parameterizations often lead to systematic biases in GCM simulations (Bennett et al., 2014; Chen et al., 2013; Muerth et 
al., 2013; Teutschbein and Seibert, 2012). Statistical bias correction is routinely applied to remove these systematic biases in GCM simulated variables before their use in either downscaling or other impact assessment models (Chen et al., 2013; Haerter et al., 2011; Johnson and Sharma, 2012; Mehrotra and Sharma, 2012). A number of bias correction approaches have been proposed in the recent past, and a detailed review of them can be found in Johnson and Sharma (2012); Watanabe et al. (2012); Lafon et al. (2013) and; Chen et al. (2013). These bias correction approaches can be broadly grouped into three main groups: mean-based, distribution-based, and distribution- and persistence-based bias correction approaches (Chen et al., 2013; Johnson and Sharma, 2012). A mean-based bias correct approach, also commonly known as delta change (Hay et al., 2000), is simple to apply; however, it cannot take into account the biases in the variability of time series which forms one of the most important attributes in any climate change impact assessment study. Distribution properties of input meteorological variables, described by the first moment (mean) and second moment (variance) or even third moment (skewness), significantly affect the hydrological process. For example, extreme hydrological events are often associated with unusual variability of rainfall and temperature. Hence, the use of a mean-based approach is not recommended if extreme values of variables are important in the impact studies, such as flood and drought (Fowler et al., 2007; Haerter et al., 2011; Mehrotra and Sharma, 2012). Consequently, more sophisticated approaches are required to

68 take into account for the biases in various distribution attributes of GCM simulated 69 variables (Pegram and Bárdossy, 2013).

70 Distribution-based bias correction approaches such as quantile mapping (Wood et al., 2004) or an improved version named equidistance quantile-matching (Li et al., 2010) can overcome this problem to some extent. Chen et al. (2013) evaluated different bias correction techniques including those belonging to the mean-based approach and those 
74 pertaining to the distribution-based approach; and concluded that distribution-based approaches are consistently better than mean-based approaches. However, as the name suggests, the commonly used distribution-based approaches only allow for the biases in the distribution of the variables to be corrected at a single specified time scale (daily, monthly or annual) overlooking the biases in the persistence of the time series. Recognising this, Johnson and Sharma (2012) proposed a nesting bias correction (NBC) approach that allows to simultaneously correct for the biases at multiple time scales. NBC is based on the assumption of a linear autoregressive model; it focuses on correcting biases in mean, standard deviation and lag-1 autocorrelation (a measure of persistence) at pre-specified time scales. As both distribution and persistence related attributes are considered in the NBC's structure, the approach falls into the category of distribution and persistence-based bias correction approaches. Johnson and Sharma (2012) tested the approach using raw GCM rainfall over Australia and concluded that NBC performed better than other bias correction approaches including the quantile mapping in correcting means, variances and lag 1 autocorrelations at pre-determined time scales if the biases were not too large. NBC was also used to bias correct the monthly GCM rainfall over India, and similar conclusions were obtained (Ojha et al., 2013). Additionally, an assessment of the implications of using NBC and other bias correction approaches for simulating future droughts over Australia was recently presented in Johnson and Sharma (2015). They concluded that in comparison to the raw GCM projections, the bias correction tends to moderate increases of the future drought frequencies, and provides smaller ranges of future changes across a large set of GCMs in many parts of Australia.

Mehrotra and Sharma (2012) extended the NBC by including more time scales and multiple recursions and called it as Recursive Nested Bias Correction (RNBC) approach. The RNBC approach included five specified time scales, namely daily, monthly, seasonal, 
99 annual and tri-annual; in addition, three to five iterations were carried out to further

100 improve the bias corrected results. The RNBC approach was tested on atmospheric

101 variables and was found to be more effective in correcting distribution and persistence

102 attributes at multiple time scales than the original NBC.

103 Recognising the importance of the low frequency variability in climate time series, bias

104 correction approaches such as NBC or RNBC are considered highly efficient as they

105 consider the biases in both distribution and persistence characteristics (lag-1 correlation) of

106 the time series at multiple selected time scales. However, the prior specification of bias

107 correction time scales and limiting to only lag-1 persistence somewhat limit their use in all

108 kind of applications.

109 These limitations of the nesting approach prompted us to look for alternatives that are

110 independent of time scales and are not limited to only lag-1 persistence. One such

111 possibility is the representation of the time series in the frequency domain. The analysis of

112 the time series in the frequency space makes it more suitable for studying the variability

113 across different time scales without the need of specifying the time scale (Bloomfield,

114 2004). In the past, Fourier transform based spectral analysis has been widely employed in

115 the fields of meteorology and hydroclimatology to examine the seasonal, annual and multi-

116 annual cycles in the time series (see Hegge and Masselink (1996); Leite and Peixoto

117 (1995); Fleming et al. (2002)). Besides, the Fourier transform technique is also applied to

118 generate surrogate time series in which linear properties of the original data are preserved

119 in the synthetic time series. More details on the application of Fourier transform technique

120 for the generation of synthetic climate time series are found in Prichard and Theiler

121 (1994); Keylock (2012); Chen et al. (2010). Following this, we propose a bias correction

122 approach that operates in the frequency space and thus negates the requirement of time 
123 scale specification as needed by the NBC and its variants. This approach is hereafter

124 referred to as the frequency-based bias correction (FBC).

125 The remainder of the paper is organised as follows. In the next section, we provide

126 theoretical and technical details of our proposed bias correction approach. In section 3, we

127 present the results of the application of FBC to GCM rainfall simulations and compare the

128 results with other bias correction approaches. In section 4, we discuss the limitations and 129 assumptions of the approach. Finally, in section 5, we summarise the results and draw the 130 conclusions.

\section{2. Methodology}

132 The idea of FBC stems from the concept that when a time series is converted in the

133 frequency domain, the variance of the time series is expressed as a function of frequency,

134 making it more suitable for studying variability at different time scales (Bloomfield, 2004).

135 Recognizing this, if a bias correction procedure is performed in the frequency domain, it is 136 expected to provide an improved representation of the observed variability across different

137 time scales including the low frequency variability, while avoiding the need for 138 specification of time scale(s) of interest. The discrete Fourier transform is one of the 139 approaches that allow the time series to be expressed in the frequency domain.

140 Transforming the time series into the frequency domain using the forward Fourier 141 transform provides information on the amplitudes and phases at different frequencies. The 142 amplitudes are used to estimate the power spectrum, also called a periodogram or Fourier 143 spectrum. As the power spectrum represents the variance of the time series as a function of

144 frequency, in FBC the power spectrum of the biased time series is modified to map that of 145 the reference time series across their respective frequencies. The bias corrected Fourier 146 spectrum is then transformed back (using an inverse Fourier transform) to the time domain 
147 to obtain bias corrected time series. This concept is explained in detail using a synthetic 148 example.

\section{2.1. The FBC logic}

150 The philosophy behind FBC is now explained by using two synthetic time series, $\mathrm{X}$ and $\mathrm{Y}$.

151 The 240 data points of these two series are obtained by adding three different sinusoidal

152 waveforms. In addition, Gaussian noise signals are added to both $\mathrm{X}$ and $\mathrm{Y}$ series. As such,

153 the total variability of $\mathrm{X}$ and $\mathrm{Y}$ is largely contributed by the three frequency components

154 with their respective amplitudes which are different for the two series. For simplicity, let 155 us assume that $\mathrm{X}$ represents the observed time series while $\mathrm{Y}$ represents the GCM 156 simulated time series; their time scales and units are arbitrary. It may be noted that the 157 mixing of variations at different time scales within one single time series makes it difficult 158 to distinguish and thus perhaps correct for biases in magnitudes of different cycles. 159 Nevertheless, it perhaps matches well with the many hydrological observations, for 160 example, rainfall which shows strong seasonal, annual and multiannual cycles.

161 These two time series are shown in the first plot of Figure 1 (plot a). An autocorrelation 162 plot provides a simple measure to examine persistence at varying time lags in a time series.

163 The other two plots of the first row of Figure 1 presents the autocorrelation plots of $\mathrm{Y}$ (plot 164 b) and $\mathrm{X}$ (plot c). Their mean and standard deviation are also shown in these plots. As can 165 be seen, the two series have different means, standard deviations and persistence statistics.

166 Plotting $\mathrm{X}$ and $\mathrm{Y}$ together as shown in Figure 1a does not tell much about the complex 167 underlying fluctuations contributing to their overall variability. Performing a routine bias 168 correction procedure on $\mathrm{Y}$ in the time domain might miss some parts of the observed (in $\mathrm{X}$ 169 series) high- and low-frequency variability and persistence in the corrected time series (as 170 shown later). To emphasize this point further, we bias correct $\mathrm{Y}$ in both time and 
171 frequency domain. In the time domain, we apply two commonly used bias correction 172 approaches. In the first approach (TBC1) we correct for the biases in the mean and 173 standard deviation of $\mathrm{Y}$ to get a bias corrected time series, Ytbcl. In the second approach 174 (TBC2), in addition to mean and standard deviation, we also correct the $\mathrm{Y}$ series for the 175 bias in lag-1 autocorrelation coefficient (Johnson and Sharma, 2012) and obtain a bias 176 corrected time series, Ytbc2. In the frequency domain, we bias correct the $\mathrm{Y}$ time series 177 using FBC (explained later in detail) and match the spectrum of $\mathrm{Y}$ to that of the $\mathrm{X}$ to 178 capture the magnitudes of different cycles in the observed series $\mathrm{X}$. The corrected 179 spectrum of $\mathrm{Y}$ together with its original phase spectrum is used for the inverse Fourier 180 transform back to the time domain to derive the corrected $\mathrm{Y}, \mathrm{Y} f b c$. The phase spectrum is 181 kept untouched because the locations of peaks or troughs with respect to the time origin of $182 \mathrm{Y}$ are not expected to shift. As both time series based bias correction approaches are 183 designed to capture first and second order moments of the observed series, it would be of 184 interest to examine how well these approaches perform in correcting for the biases in the 185 persistence attributes. Plots $\mathrm{d}$, e and $\mathrm{f}$ of Figure 1 (bottom row) present autocorrelation 186 plots of the bias corrected time series Y $t b c 1, \mathrm{Y} t b c 2$ and Y $f b c$, respectively. As TBC1 187 corrects for the biases in mean and standard deviation, the autocorrelation structure 188 remains unaffected owing to the fact that correcting for means and standard deviation does 189 not change the temporal autocorrelation structure of the time series (plot d, Figure 1). 190 TBC2, in addition to mean and standard deviation also removes the biases in the lag-1 191 correlation (built in the model structure), although biases at other lags are still present (plot 192 e, Figure 1). The FBC reproduces the exact observed autocorrelation plot (plot f, Figure 1).

193 Although it is possible to correct for the biases in the higher order lags in the time domain 194 by using a more complex bias correction procedure, it will introduce added mathematical 195 complexity and would correct for the specified lags only. Converting the time cycles in 
196 frequencies allows one to apply a simple addition/subtraction procedure to correct for the

197 biases in these frequencies and therefore, provides a simple yet powerful tool to correct for

198 the biases in all dominant cycles present in the time domain without involving additional

199 complexities in the bias correction procedure.

\subsection{FBC Formulation}

201 The formulation of FBC is based on the discrete Fourier transform technique. Appendix A 202 provides the mathematical basis for the discrete Fourier transform technique and 203 estimation of power spectrum based on the Fourier components. For clarity, the parameters 204 of the FBC bias-correction approach are estimated using observed and calibration period data sets and these are applied to an independent data set (validation period or future period) not used in the calibration process. The following describes the step-wise procedure used to implement the FBC algorithm.

(i) Preprocess the raw data before the actual application of the bias correction approach by removing seasonality or trends, if any, present in the data. A majority of hydrology-relevant variables, such as precipitation, exhibit strong seasonal variations. Removing seasonality is necessary when data exhibit multiple seasonal patterns (i.e. winter and summer patterns). These differing seasonal variations are removed by using monthly statistics (i.e. means, standard deviations and lag-1 correlations) derived from the observed and calibrated period datasets (Johnson and Sharma, 2012; Sharma et al., 2013). Furthermore, de-trending is necessary for atmospheric variables, which are known to exhibit significant systematic trends, for example, temperature (Brooks, 2011; Johnson and Sharma, 2012). The deseasonalising and de-trending of time series transform the data to be closer to the stationarity conditions assumed, a prerequisite for the application of Fourier 
transform. These pre-processed data sets are called as the observed, calibration and validation residuals.

(ii) Transform observed, calibration and validation residuals into the frequency domain using the discrete Fourier transform technique as presented in the Appendix A. Calculate amplitudes and phases across different frequencies of the observed, calibration and validation residuals using equation $A 5$ and $A 6$. Let $A^{\text {obs }}(f), A^{m c}(f)$, and $A^{\mathrm{mf}}(\mathrm{f})$ represent the amplitudes of the observed, calibration and validation residuals at frequency f, respectively. Likewise, $\phi^{\mathrm{obs}}(\mathrm{f}), \phi^{\mathrm{mc}}(\mathrm{f})$, and $\phi^{\mathrm{mf}}(\mathrm{f})$ represent the phases of the observed, calibration and validation residuals at frequency $\mathrm{f}$, respectively.

(iii) Derive the (Fourier) power spectra of the observed, calibration and validation residuals from their respective amplitude spectra using equation A9a and A9b. Denote $\mathrm{FP}^{\mathrm{oc}}, \mathrm{FP}^{\mathrm{mc}}$, and $\mathrm{FP}^{\mathrm{mf}}$ as the power spectra of the observed, calibration and validation residuals, respectively.

(iv) Map the power spectrum of the calibration residuals onto that of the observed residuals of the same control period in the frequency domain. This is implemented by taking the difference of the periodogram ordinates of $\mathrm{FP}^{\mathrm{oc}}$ and those of $\mathrm{FP}^{\mathrm{mc}}$ at the corresponding frequencies. This difference is the so-called spectral fitcoefficients. Add the spectral fit-coefficients to the periodogram ordinates of $\mathrm{FP}^{\mathrm{mc}}$ at the corresponding frequencies to yield the corrected Fourier spectrum of the calibration residuals $\left(\widetilde{F P}^{m c}\right)$ to match exactly with the Fourier spectrum of the observed residuals.

(v) Apply spectral fit-coefficients obtained in step (iv) to bias correct for the power spectrum of the validation residuals. Specifically, add the spectral fit-coefficients to 
$\mathrm{FP}^{\mathrm{mf}}$ at the corresponding frequencies to produce the bias corrected power spectrum of the validation residuals $\left(\widetilde{F P}^{m f}\right)$.

(vi) Once $\widetilde{F P}^{m c}$ and $\widetilde{F P}^{m f}$ are obtained, rearrange equations $\mathrm{A} 9 \mathrm{a}$ and $\mathrm{A} 9 \mathrm{~b}$ to calculate amplitudes across the different frequencies. Denote $\tilde{A}^{m c}(f)$ and $\tilde{A}^{m f}(f)$ as the amplitudes at frequency $f$ derived from the corresponding $\widetilde{F P}^{m c}$ and $\widetilde{F P}^{m f}$.

(vii) Construct the modified Fourier components using $\tilde{A}^{m c}(f)$ and $\phi^{\mathrm{mc}}(\mathrm{f})$ for the calibration residuals, and $\tilde{A}^{m f}(f)$ and $\phi^{\mathrm{mf}}(\mathrm{f})$ for the validation residuals (equation A7) up to the Nyquist frequency $\left(f_{c}\right)$. Recall that the Fouier components at the frequencies greater than $f_{c}$ are just the complex conjugate of those at the frequencies less than $f_{c}$ (Hegge and Masselink, 1996).

254 (viii) Once the modified complex Fourier components of the calibration and validation

(ix) In the last step, rescale back the bias corrected calibration and validation residuals following a reversed deseasonalising procedure adopted in the step (i) using the observed statistics (i.e. observed means, standard deviations and/or lag-1 correlations) as described in Sharma et al. (2013) to obtain the bias corrected time series for calibration and validation periods.

\section{Application of FBC on Raw GCM Precipitation}

\subsection{Data}

265 The observed and GCM simulated monthly rainfall time series are used to assess the

266 performance of FBC. The observed rainfall series used in this study is monthly gridded

267 rainfall on a $0.5^{\circ}$ latitude - longitude resolutions for the period from 1901 to 2000 , 
provided by the Australian Bureau of Meteorology (BOM). This dataset covers the whole land mass of Australia, from $10^{\circ} \mathrm{S}$ to $45^{\circ} \mathrm{S}$ latitude and from $115^{\circ} \mathrm{E}$ to $165^{\circ} \mathrm{E}$ longitude.

270 Simulated monthly raw precipitation of MIROC5 GCM is extracted from the Coupled

271 Model Intercomparison Project phase 5 (CMIP5), archived by the Program for Climate

272 Model Diagnosis and Intercomparison (PCMDI) website: http://pcmdi9.1lnl.gov/esgf-web-

273 fe/live. The MIROC5 is maintained by the Atmosphere and Ocean Research Institute (The

274 University of Tokyo), National Institute for Environmental Studies, and Japan Agency for

275 Marine-Earth Science and Technology. It is noteworthy that we use MIROC5 to illustrate

276 the proposed approach; however, the proposed bias corrected approach here is generic and

277 can be applied (and has been applied though results are not presented for conciseness) to

278 other GCMs as well.

279 MIROC5 simulated rainfall has a grid resolution of approximately $1.4^{\circ}$ latitude and 280 longitude; therefore, a common $1.0^{\circ}$ rectangular grid is chosen, and the MIROC simulated 281 and observed rainfall time series is re-gridded into the same spatial resolution using 282 bilinear interpolation. As a result, there are 772 grid points $\left(1.0^{\circ}\right.$ latitude and longitude 283 resolutions) covering the Australian land surface. Each grid point has single monthly time 284 series from the observed and simulated monthly precipitation over the period from 1901 to 285 2000. Like most of the bias correction approaches, the proposed bias correction procedure 286 is also performed independently at each grid cell assuming spatial independence (Johnson 287 and Sharma, 2012).

\subsection{Methodology Assessment}

289 The performance of FBC is assessed in cross validation by dividing the available 100 years 290 of record into five 20 years long non-overlapping subsets. Each subset is then used as a 291 validation set (20-year period), with the remaining data (80-year period) used as a 
292 calibration set. It is important to note that unequal length of the calibration and validation 293 datasets also results in dissimilar frequencies while deriving the Fourier spectra. To deal 294 with this issue, in the step (ii) of the FBC procedure, we construct averaged periodogram 295 estimates for the observed and current GCM residuals having the same frequencies as 296 those defined by the validation dataset (more information is presented in the discussion 297 section).

298 In order to have a proper assessment of the performance of FBC, we also apply the 299 empirical quantile mapping (EQM) and the recursive nesting bias correction (RNBC) 300 approaches and compare the results. Going by the model structure, we know that EQM 301 corrects for the distribution biases only at a specified time scale and does not correct for 302 the biases in the temporal persistence. The bias correction formulation for EQM is based 303 on equation 1 following Li et al. (2010) with the approach applied separately for each 304 month. $\tilde{x}_{m f}=x_{m f}+\left\{F_{o c}^{-1}\left(F_{m f}\left(x_{m f}\right)\right)-F_{m c}^{-1}\left(F_{m f}\left(x_{m f}\right)\right)\right\}$

306 In which, $x$ is a climate variable, $F($.$) is the empirical cumulative distribution of either$ 307 observation $(o)$ or model $(m)$ for the calibration period $(c)$ or future period $(f)$.

308 The RNBC as described in Mehrotra and Sharma (2012), corrects for distribution as well 309 as lag-1 persistence attributes at the predefined time scales. In the present study, RNBC is 310 applied at monthly and annual time scales.

311 The performance of these approaches is assessed on the basis of reproduction of various 312 distribution- and persistence-based attributes. For the distribution-based properties, we use 313 means and standard deviations at monthly, seasonal and annual time scales. For the 314 persistence-based attributes, we measure the persistence of oscillations within the time series using the autocorrelation function (ACF). We use the Root-Mean-Squared Error 
316 (RMSE) statistic (Equation 2) as an error measure between the ACF of the monthly

317 observed time series and that of the monthly simulated time series.

$318 R M S E_{A C F}=\sqrt{\frac{\sum_{l=1}^{L}\left(\rho_{o, \tau}-\rho_{m, \tau}\right)^{2}}{L}}$

319 Where, $L$ is the number of autocorrelation lags used and $\rho_{\mathrm{o}, \tau}$ and $\rho_{\mathrm{m}, \tau}$, respectively, are the

320 sample autocorrelation functions at lag $\tau$ for the observed and simulated time series. It is

321 known that when $L$ increases, the significance of $\rho_{\mathrm{o}, \tau}$ and $\rho_{\mathrm{m}, \tau}$ decreases; therefore,

322 following Caiado et al. (2009), we adopt $L=\mathrm{N}_{\text {data }} / 10$, where $\mathrm{N}_{\text {data }}$ is the number of data

323 points.

324 To measure how well the bias-corrected GCM precipitation time series capture observed

325 low-frequency variability, we use aggregated persistence score (APS), proposed by

326 Rocheta et al. (2014). In general, the APS score is an alternative metric of persistence,

327 related to the Hurst exponent; however, it measures the magnitude of differences between

328 the GCM and observed standard deviations scaled by no-persistence confidence interval of

329 the standard deviations over all aggregation periods (Rocheta et al., 2014). The procedure

330 for calculating APS involves two steps: (i) obtain the scaled deviations at different

331 aggregated periods (denotes as $\mathrm{SD}_{i}$ ) by dividing the differences in standard deviations of

332 the observed and simulated time series at each aggregated period by a scaling factor

333 (equation 3 ) and (ii) average $\mathrm{SD}_{i}$ over all the aggregated periods (equation 4).

$334 S D_{i}=\frac{A_{i}}{B_{i}}=\frac{S(i)_{m}-S(i)_{o}}{B_{i}}$

$335 \quad A P S=\frac{1}{i} \sum_{i} S D_{i}$

336 Where $\mathrm{SD}_{i}$ is the scaled deviance at aggregation period $i$; $\mathrm{S}(i)$ is the standard deviation of 337 the $i^{\text {th }}$ aggregation period for the GCM simulations $(m)$ or the observations $(o)$; and $\mathrm{B}_{i}$ is 
the reference no-persistence confidence interval of the standard deviation for the same aggregation period $i$.

340 For spatial averaging of the APS values over all grid cells, Rocheta et al. (2014) defined

341 three kinds of spatially averaged APS metrics: (1) APS $_{a b s}$ measuring the overall

342 performance across the land mass in term of reproducing the persistence without

343 considering over- or under-simulations of the persistence (equation 5); (2) $\mathrm{APS}_{\text {pos }}$

344 measuring an over-simulation of the persistence over the land mass (equation 6); and (3)

$345 \mathrm{APS}_{n e g}$ measuring the persistence under-simulation over the study area (equation 7).

$346 \quad A P S_{a b s}=\frac{1}{n} \sum_{n} \frac{1}{i} \sum_{i}\left|S D_{i}\right|$

$347 \quad A P S_{\text {pos }}=\frac{1}{n} \sum_{n} \frac{1}{i} \sum_{i} S D_{i}^{+}$

$348 \quad A P S_{n e g}=\frac{1}{n} \sum_{n} \frac{1}{i} \sum_{i} S D_{i}^{-}$

349 Where $\left|S D_{i}\right|, S D_{i}^{+}, S D_{i}^{-}$the absolute, positive and negative values of the scaled deviance 350 at aggregation period $i$ and $n$ is the number of grid cells.

351 In addition, the 3 year and 5-year minimum rainfall totals are also considered in order to assess the performance of the approaches in capturing runs of low rainfall years.

\subsection{Results}

354 The performance of EQM, RNBC and FBC approaches is evaluated in terms of reproduction of observed distribution and persistence attributes in the cross validation

356 period across a range of time scales of interests. The seasonal time series is formed by 357 accumulating monthly rainfall values for the four seasons, namely; autumn (March, April and May), winter (June, July and August), spring (September, October, and November) and summer (December, January, and February). The annual rainfall time series is formed by summing up the twelve monthly rainfall values of each calendar year. We first look at 
361 how well the three bias correction approaches perform in reproducing the mean and 362 standard deviation, which to a large extent are representative of the distributional 363 attributes. Figure 2 presents the scatter plots of observed, raw and bias corrected monthly, 364 seasonal and annual means and standard deviations at individual grid points. In Figure 2, 365 each panel contains three plots showing monthly, seasonal and annual statistics. Dots in 366 these plots indicate grid points. It can be seen that the GCM raw simulations misrepresent 367 the observed means and standard deviations (column 1 of Figure 2). The model overpredicts means and standard deviations for most of the grid cells over Australia across the

369 three time scales. As, after performing the bias correction at monthly time scale, mean 370 biases at all other time scales are automatically taken care of; results for the mean at 371 annual time scale only are presented (the three last columns in the top row in Figure 2).

372 For the second-order moment, represented here by the standard deviation (SD), all the 373 approaches perform well at the monthly time scale (bottom row of Figure 2). However, at 374 seasonal and annual time scales, EQM shows some scatter in comparison to RNBC and 375 FBC. As EQM corrects for the biases in the distribution attributes at monthly time scales 376 only, the biases in the variations at other time scales are not accounted for (Haerter et al., 377 2011). For RNBC, the annual standard deviation is an explicitly defined parameter while 378 in FBC corrects separately for each variation components associated with each frequency 379 in the frequency domain.

380 It is recalled that the main strength of our proposed approach lies in its capability to correct

381 for the biases in persistence attributes. We present next the performance evaluation of bias 382 correction approaches in terms of their ability to correct for the biases in persistence based 383 attributes.

384 Box plots in Figure 3 present the distribution of the prediction error for $\mathrm{RMSE}_{A C F}$ 385 (equation 2) across all grid cells over Australia. It can be seen that FBC outperforms 
RNBC and EQM with the lowest mean error and the least spatial variation for $\mathrm{RMSE}_{A C F}$

387 (Figure 3). This is due to the fact that the FBC technique corrects for magnitudes of the various kinds of oscillations associated with different frequencies separately in the frequency domain so that the magnitudes of oscillations in the observed time series are well reproduced. Although RNBC corrects for the biases in lag-1 autocorrelation at monthly and annual time scales, both RNBC and EQM perform similarly in the RMSE $\mathrm{ACF}_{\mathrm{A}}$ statistic (Figure 3). It appears that the $\mathrm{RMSE}_{A C F}$ statistic is influenced by the correlations at higher lags used in the calculation of ACF .

We now specifically look at the RMSE of autocorrelations at some significant lags (normally linked to stronger persistence), for example, lag-1, lag-2 and lag-3 autocorrelations of the monthly and annual time series averaged over all grid points (Table 1). While RNBC approach has the least RMSE for lag-1 autocorrelations at monthly and annual time scales (an integral part of the model structure), FBC does for other lags and time scales (Table 1). These results are quite encouraging as FBC corrects for the biases at multiple lags and time scales.

401 Figure 4 presents the APS scores, calculated using temporal aggregations from 24 to 60 402 months with a time step of one month at each grid cell over Australia. We consider the 403 APS value within the range of -0.25 to 0.25 to be close to zero to calculate the percentage 404 of grid cells showing the over-simulation or under-simulation of rainfall persistence 405 (Rocheta et al., 2014). It can be seen that the raw model simulations over-estimate the 406 persistence over most of the Australian landmass, with $89 \%$ of grid cells showing the over407 simulation and $6 \%$ of grid cells showing the under-simulation (Figure 4a). The bias 408 corrected simulations from EQM, RNBC and FBC show improvements over raw GCM 409 simulations in capturing the persistence with a significant reduction of over-simulation of 410 persistence as shown in Figures $4 b$, c, and d. Considering the overall performance in terms 
411 of reproduction of observed persistence and ignoring the over-simulation or under-

412 simulation (APS ${ }_{a b s}$ ), FBC provides the best performance with $\mathrm{APS}_{a b s}$ of 0.41 as opposed to

4130.62 and 0.59 for the EQM and RNBC approaches, respectively.

414 We finally compare the performance of the three approaches in representing the

415 interannual variability expressed in terms of the runs of low rainfall years by considering

416 the 3 and 5 year minimum total rainfalls rescaled by the observed annual standard

417 deviations (Johnson and Sharma, 2012) (Figure 5). Although all the approaches provide a

418 good simulation of these statistics, FBC provides the lowest RMSE over Australia for both

419 the 3-year and 5-year minimum total rainfalls (Figure 5).

\section{4. Discussion}

421 Bias correction approaches are routinely applied to remove the systematic biases in GCM

422 outputs. Proper reproduction of observed year-to-year or low frequency variability in

423 GCM output of climate variables has received considerable attention in the recent past 424 with quite a few studies concentrating on the issue of appropriate bias correction approaches for use in hydrological impact studies (Chen et al., 2013; Johnson and Sharma, 2011; Li et al., 2010; Rocheta et al., 2014). Use of a proper bias correction approach assumes more importance for countries like Australia, where the climate is frequently

428 characterized by cycles of below and above average rainfall leading to severe droughts and widespread flooding (Westra and Sharma, 2006). The strength of FBC approach lies in its capability of working in the frequency domain and correcting for the magnitudes of

431 different fluctuations across multiple frequencies (they are independent of time origin and 432 time scale).

433 Like any other bias correction approaches, there are some limitations and assumptions associated with the FBC approach as well. For example, the selection of data length might 
435 affect the ability of representing oscillation components in the frequency domain. Fourier 436 frequencies including the lowest frequency (fundamental frequency), harmonic frequencies

437 and the highest frequency (Nyquist frequency) depends on the time period of data 438 (Appendix A). Therefore, the amplitudes of oscillations obtained by the Fourier transform, 439 which are gauged at the harmonic frequencies up to the Nyquist frequency, also depend on 440 the data length. The FBC approach cannot precisely correct for biases in the amplitudes at 441 frequencies other than the harmonic frequencies defined by the data length.

442 When the calibration and validation (or future) time series have unequal lengths, Fourier 443 frequencies at which periodogram ordinates are gauged are not the same(Caiado et al., 444 2009). Therefore, before applying the bias correction procedure in the frequency domain, 445 either one has to drop the extra data points and make both series to be of the same length 446 or somehow calculate the periodogram ordinates at common frequencies. If time series of 447 the calibration period is long enough to be divided into multiple segments of data length 448 equal to the future time series, one of the solutions is to calculate the periodogram of each 449 segment separately and construct an averaged periodogram to have the same frequencies 450 as that of the validation (or future) time series (this procedure is also implemented in 451 section 3). It is noteworthy that the averaged periodogram also provides statistically 452 reliable and stable Fourier spectral estimates in comparison to single time series 453 periodogram (Hegge and Masselink, 1996; Thomson and Emery, 2014). If the data length 454 is not long enough to be divided into multiple segments, an alternative solution is to use 455 the interpolation periodogram technique proposed by Caiado et al. (2009). In this 456 approach, the periodogram ordinates at common frequencies are obtained by interpolation. 457 In order to get a better idea of the implication of these alternatives on the final bias 458 corrected time series, in Appendix B, we present a few common statistics of interest 
459 obtained by using the averaged and interpolated periodogram alternatives of the FBC 460 approach.

461 In line with the other commonly used bias correction approaches, the FBC approach also

462 assumes that biases in the magnitudes of different variations of the control period are same

463 as those of the future period. Although this assumption simplifies the bias correction 464 procedure, it may not necessarily be hold true if biases in the amplitudes of oscillations evolve over time. This limitation can be overcome by investigating the spectral behaviour 466 of time series evolving over time using a time-frequency analysis such as dynamic Fourier 467 analysis or Wavelet analysis (Shumway and Stoffer, 2005). Future work could investigate 468 the utility of the time-frequency analysis for this purpose.

469 The FBC approach, being univariate, does not account for the dependence biases across 470 climate variables. We know that many climate variables have strong physical relationships 471 leading to strong cross-dependence structure, for example, temperature and rainfall. A 472 univariate bias correction approach may alter the physical consistency of multiple climate 473 variables, of importance in applications such as downscaling or other hydrological 474 applications (Mehrotra and Sharma, 2010; Mehrotra and Sharma, 2015). An extension of 475 the univariate FBC to the multivariate case is an interesting research problem which we 476 intend to take up in the near future.

477 The FBC approach as presented here operates independently at individual grid cells; this 478 means the approach does not take into account the observed spatial dependence - a 479 common practice followed in the most bias correction approaches. In many applications, 480 preserving the spatial correlation structure is very important (for example see Johnson and 481 Sharma (2012)). Some ideas for correcting for the biases in spatial dependence are 
482 discussed in Johnson and Sharma (2012); Mehrotra and Sharma (2015). It is possible to 483 extend the FBC following these ideas.

484 The paper illustrates the utility of the FBC approach using monthly data. Monthly records 485 are useful in some applications such as drought or water resources planning and 486 assessment whereas daily records are required for other applications such as flood studies 487 and streamflow modelling (Johnson and Sharma, 2012). In order to apply the FBC 488 approach to daily data, the pre-processing step (step 1 of section 2.2., FBC Formulation) 489 has to be suitably modified to accommodate the daily variations. For example, a treatment 490 for seasonality for daily data can be found in Rajagopalan and Lall (1999) and Mehrotra 491 and Sharma (2015). In addition, some kinds of smoothing techniques would also be

492 needed to filter out the noise from the signals, if daily data spectra are too noisy (Chatfield, 493 2003). Future work will address these issues.

494 The applicability of the proposed approach is demonstrated using a single GCM (MIROC) 495 and variable (rainfall). The methodology presented here, however, is generic and can be 496 applied to any GCMs or other climate variables commonly used in statistical/dynamical 497 downscaling and hydrology. We have successfully applied the proposed approach to the 498 precipitation output of 37 GCMs (results available upon request) and have found improved 499 performance of the FBC approach over other two approaches used.

\section{5. Conclusions}

501 This work presents a new bias correction procedure that operates in the frequency domain.

502 The FBC utilises the Fourier transform technique to convert the series from time to the 503 frequency domain and then corrects for the biases in magnitudes of different fluctuations 504 separately, which is not possible using a routine bias correction approach in the time 
505 domain. We believe that this research provides a new direction for conducting further

506 research in improving the quality of raw GCM outputs.

507 The performance of the approach is compared and assessed using raw GCM monthly

508 rainfall simulations in cross validation and the two existing bias correction approaches,

509 namely an empirical quantile mapping (EQM) and a recursive nesting bias correction

510 (RNBC). The results suggest that while the performance of FBC is comparable with other

511 two approaches in terms of reproduction of the observed first- and second- moment

512 statistics, it outperforms in reproducing various persistence based attributes at multiple

513 time scales. As a result, it offers a great promise for use in various impact assessment

514 applications, particularly in hydrological impact studies where year to year variability

515 plays an important role.

\section{Appendix A: Discrete Fourier Transform and Fourier Spectrum Estimation}

517 The discrete Fourier transform of a finite time series $\mathrm{x}(\mathrm{n})$ of $\mathrm{N}$ data points taken at regular

518 intervals of time $\Delta \mathrm{t}$ (the forward transform) can be expressed as follows (Hegge and

519 Masselink, 1996):

$520 \quad \mathrm{X}(\mathrm{k})=\sum_{\mathrm{n}=0}^{\mathrm{N}-1} \mathrm{x}(\mathrm{n}) \mathrm{e}^{-\mathrm{jn} \omega_{\mathrm{k}}}$

521 in which:

$522 \mathrm{k}=$ number of cycles (per time period $\mathrm{P}=\mathrm{N} \Delta \mathrm{t}$ ) or so-called harmonic numbers, $\mathrm{k}=0,1, \ldots$,

523 N-1. Harmonic $1(\mathrm{k}=1)$ corresponds to one cycle over the time period, and higher 524 harmonics are integer multiples of one cycle over the time period.

$525 \mathrm{X}(\mathrm{k})=$ Fourier components or coefficients at the frequencies $\mathrm{f}_{k}$ or $\omega_{k}$

$526 \omega_{k}=2 \pi f_{k}=\frac{2 \pi k}{N \Delta t}$

$527 \omega_{k}=$ frequency of the $\mathrm{k}^{\text {th }}$ Fourier coefficient in radians per unit time 
$528 \mathrm{f}_{k}=$ frequency of the $\mathrm{k}^{\text {th }}$ Fourier coefficient in cycles per unit time, it is also called 529 harmonic frequency.

$530 \quad \mathrm{f}_{k}=\frac{\mathrm{k}}{\mathrm{N} \Delta \mathrm{t}}$

531 The Fourier coefficients are complex, each having a real $\left(\mathrm{X}_{\text {real }}\right)$ and imaginary part $\left(\mathrm{X}_{\text {imag }}\right)$;

532 therefore, we can represent equation A1 in the below form (Fleming et al., 2002):

$533 \quad \mathrm{X}(\mathrm{k})=\mathrm{X}_{\text {real }}(\mathrm{k})+\mathrm{j} \mathrm{X}_{\text {imag }}(\mathrm{k})$

534 These complex Fourier coefficients $X(k)$ are then used to calculate the amplitude $A(k)$ and 535 phase $\phi(\mathrm{k})$ (with respect to the beginning the series) for each $\mathrm{k}^{\text {th }}$ component as follows 536 (Fleming et al., 2002):

$537 \mathrm{~A}(\mathrm{k})=\left(\mathrm{X}_{\text {real }}^{2}(\mathrm{k})+\mathrm{X}_{\text {imag }}^{2}(\mathrm{k})\right)^{1 / 2}$

$538 \theta(\mathrm{k})=\tan ^{-1}\left[\mathrm{X}_{\text {imag }}(\mathrm{k}) / \mathrm{X}_{\text {real }}(\mathrm{k})\right]$

539 Equation A1 may be redefined in terms of the amplitude $\mathrm{A}(\mathrm{k})$ and phase $\phi(\mathrm{k})$ as follows

540 (Fleming et al., 2002):

$541 \quad \mathrm{X}(\mathrm{k})=\mathrm{A}(\mathrm{k}) \mathrm{e}^{\mathrm{j} \theta(\mathrm{k})}$

542 In summary, the forward discrete Fourier transform allows the time series to be 543 represented in the frequency domain by the Fourier components whose frequencies are 544 integer multiples of $(\mathrm{N} \Delta \mathrm{t})^{-1}$ (fundamental frequency), ranged from a frequency of zero $\left(\mathrm{f}_{\mathrm{o}}\right)$ 545 to Nyquist frequency $\left(f_{c}\right)$. The Fourier component at the frequency of zero $\left(f_{0}\right)$ is equal to 546 the mean of the time series (Fleming et al., 2002). The fundamental frequency $(\mathrm{N} \Delta \mathrm{t})^{-1}$ is

547 the lowest frequency which can be detected by the data series using the discrete Fourier 548 transform, also defined as the frequency bandwidth (Hegge and Masselink, 1996). The 549 Nyquist frequency $\left(\mathrm{f}_{c}=\mathrm{f}_{N / 2}=1 / 2 \Delta \mathrm{t}\right)$ is the highest frequency which the Fourier transform 
550 can resolve by the given time series (Hegge and Masselink, 1996). The Fourier 551 components at the frequencies greater than $\mathrm{f}_{c}$ are just the complex conjugate of those at the

552 frequencies less than $\mathrm{f}_{c}$ (Hegge and Masselink, 1996).

553 The inverse discrete Fourier transform, which allows the Fourier components from the

554 frequency domain back to the time domain, is defined using equation A8 (Thomson and

555 Emery, 2014) :

556

$\mathrm{x}(\mathrm{n})=\frac{1}{\mathrm{~N}} \sum_{\mathrm{k}=0}^{\mathrm{N}-1} \mathrm{X}(\mathrm{k}) \mathrm{e}^{\mathrm{jn} \omega_{\mathrm{k}}}$

557 In practice, the implementation of the forward and inverse discrete Fourier transforms can

558 be achieved by fast Fourier transform (FFT) and inverse fast Fourier transform (IFFT)

559 algorithms of the programming language, Matlab (Hegge and Masselink, 1996).

560 After obtaining the Fourier components, the one-sided Fourier spectrum or power

561 spectrum (also called periodogram) can be calculated as follows (Hegge and Masselink, 562 1996):

$563 \quad F P(k)=\frac{A(k)^{2}}{N}$ for $\mathrm{k}=0, \frac{N}{2}$

$564 F P(k)=\frac{2 A(k)^{2}}{N}$ for $\mathrm{k}=1, \ldots \frac{N}{2}-1$

565 To improve the statistical reliability of the periodogram estimate, it is recommended to 566 combine periodograms estimates obtained from different segments of the long time series 567 using segment-averaging technique (referred to Hegge and Masselink (1996); Thomson 568 and Emery (2014)). The periodogram estimate obtained from the segment-averaging 569 technique are called the average periodogram.

570 Appendix B: Compares a few common statistics of the bias corrected results obtained 571 by using averaged periodogram (called FBCa) and interpolation periodogram techniques (called FBC $i$ ). 
573 Table B.1. RMSE of statistics of interest and the APS metric over Australia based on the

574 raw GCM time series and bias corrected time series using $\mathrm{FBC} a$ and $\mathrm{FBC} i{ }^{*}$

\begin{tabular}{llll}
\hline Statistics & Raw GCM & FBC $a$ & FBC $i$
\end{tabular}

Monthly Standard Deviation (mm)

Annual Mean (mm)

Annual SD (mm)

3 Year Minimum Total Rainfall (mm)

5 Year Minimum Total Rainfall (mm)

Mean of RMSE for ACF*

Standard Deviation of RMSE for ACF*

Monthly Lag-1 Autocorrelation

Monthly Lag-2 Autocorrelation

Monthly Lag-3 Autocorrelation

Annual Lag-1 Autocorrelation

Annual Lag-2 Autocorrelation

Annual Lag-3 Autocorrelation

$\mathrm{APS}_{a b s}$
28.26

420.18

72.34

1004.

1743

0.062

0.027

0.075

0.061

0.070

0.139

0.140

0.171

1.92
1.57

31.09

12.80

0.8

1.34

1.5

0.028

0.033

0.006

0.006

0.032

0.033

0.027

0.038

0.029

0.039

0.115

0.130

0.107

0.131

0.128

0.140

* RMSE for ACF is calculated using equation 2. The best results are highlighted in bold.

575 The results presented in Table B.1 suggest that although both the techniques reduce the

576 biases in various statistical attributes, the results of averaging periodogram technique

$577(\mathrm{FBC} a)$ are better in comparison to the interpolation periodogram technique. These results 
confirm the earlier findings that the averaged periodogram provides statistically reliable and stable Fourier spectral estimates in comparison to single time series periodogram

580 (Hegge and Masselink, 1996; Thomson and Emery, 2014). However, in most practical 581 situations, the data length is not long enough to calculate the average periodogram and the 582 interpolation periodogram technique provides an easy workable solution to deal with 583 unequal data length situations.

\section{Acknowledgments}

585 We acknowledge the World Climate Research Programme's Working Group on Coupled 586 Modelling which is responsible for CMIP; the climate modeling groups (namely for this study, Atmosphere and Ocean Research Institute (The University of Tokyo), National Institute for Environmental Studies, and Japan Agency for Marine Earth Science and Technology) for producing and making available their model output; and the Program for

590 Climate Model Diagnosis and Intercomparison (PCMDI) for collecting and achieving the model data. For CMIP the U.S. Department of Energy's Program for Climate Model Diagnosis and Intercomparison provides coordinating support and led the development of software infrastructure in partnership with the Global Organization for Earth System

594 Science Portals. The gridded observed precipitation for Australia was provided by the 595 Australian Bureau of Meteorology (BOM). The first author would like to acknowledge the 596 financial support from the Austrailian Awards Scholarships (AAS). We would also like to 597 thank the editor, associate editor, Geoff Pegram and the two anonymous reviewers for their 598 constructive comments that helped us to improve the manuscript.

\section{References}

600 Bennett, J.C. et al., 2014. Performance of an empirical bias-correction of a high-resolution 601 climate dataset. International Journal of Climatology, 34(7): 2189-2204. 602 DOI:10.1002/joc.3830 
Bloomfield, P., 2004. Fourier analysis of time series: an introduction. John Wiley \& Sons. Fourier Transforms-New Analytical Approaches and FTIR Strategies, Goran Nikolic (Ed.), ISBN: 978-953.

Caiado, J., Crato, N., Peña, D., 2009. Comparison of times series with unequal length in the frequency domain. Communications in Statistics-Simulation and Computation ${ }^{\circledR}, 38(3)$ : 527-540.

Chatfield, C., 2003. The Analysis of Time Series: An Introduction, Sixth Edition (Chapman \& Hall/CRC Texts in Statistical Science). Chapman and Hall/CRC. DOI:citeulike-article-id: 1147733

Chen, J., Brissette, F.P., Chaumont, D., Braun, M., 2013. Finding appropriate bias correction methods in downscaling precipitation for hydrologic impact studies over North America. Water Resources Research, 49(7): 4187-4205. DOI:10.1002/wrcr.20331

Chen, J., Brissette, F.P., Leconte, R., 2010. A daily stochastic weather generator for preserving low-frequency of climate variability. Journal of Hydrology, 388(3-4): 480-490. DOI:http://dx.doi.org/10.1016/j.jhydrol.2010.05.032

Fleming, S.W., Marsh Lavenue, A., Aly, A.H., Adams, A., 2002. Practical applications of spectral analysis to hydrologic time series. Hydrological Processes, 16(2): 565-574. DOI:10.1002/hyp.523

Fowler, H.J., Blenkinsop, S., Tebaldi, C., 2007. Linking climate change modelling to impacts studies: recent advances in downscaling techniques for hydrological modelling. International Journal of Climatology, 27(12): 1547-1578. DOI:10.1002/joc. 1556

Haerter, J.O., Hagemann, S., Moseley, C., Piani, C., 2011. Climate model bias correction and the role of timescales. Hydrol. Earth Syst. Sci., 15(3): 1065-1079. DOI:10.5194/hess-15-1065-2011

Hay, L.E., Wilby, R.L., Leavesley, G.H., 2000. A comparison of delta change and downscaled GCM scenarios for three mountainous basins in the United State. JAWRA Journal of the American Water Resources Association, 36(2): 387-397. DOI:10.1111/j.1752-1688.2000.tb04276.x

Hegge, B.J., Masselink, G., 1996. SPECTRAL ANALYSIS OF GEOMORPHIC TIME SERIES: AUTO-SPECTRUM. Earth Surface Processes and Landforms, 21(11): 1021-1040. DOI:10.1002/(SICI)1096-9837(199611)21:11<1021::AIDESP703>3.0.CO;2-D

Johnson, F., Sharma, A., 2009. Measurement of GCM Skill in Predicting Variables Relevant for Hydroclimatological Assessments. Journal of Climate, 22(16): 43734382. DOI:10.1175/2009JCLI2681.1

Johnson, F., Sharma, A., 2011. Accounting for interannual variability: A comparison of options for water resources climate change impact assessments. Water Resources Research, 47(4): W04508. DOI:10.1029/2010WR009272

Johnson, F., Sharma, A., 2012. A nesting model for bias correction of variability at multiple time scales in general circulation model precipitation simulations. Water Resources Research, 48(1): W01504. DOI:10.1029/2011WR010464

Johnson, F., Sharma, A., 2015. What are the impacts of bias correction on future drought projections? Journal of Hydrology, 525: 472-485. DOI:http://dx.doi.org/10.1016/j.jhydrol.2015.04.002

Keylock, C.J., 2012. A resampling method for generating synthetic hydrological time series with preservation of cross-correlative structure and higher-order properties. Water Resources Research, 48(12): W12521. DOI:10.1029/2012WR011923 
Lafon, T., Dadson, S., Buys, G., Prudhomme, C., 2013. Bias correction of daily precipitation simulated by a regional climate model: a comparison of methods. International Journal of Climatology, 33(6): 1367-1381. DOI:10.1002/joc.3518

Leite, S.M., Peixoto, J.P., 1995. Spectral analysis of climatological series in Duero Basin. Theor Appl Climatol, 50(3-4): 157-167. DOI:10.1007/BF00866114

Li, H., Sheffield, J., Wood, E.F., 2010. Bias correction of monthly precipitation and temperature fields from Intergovernmental Panel on Climate Change AR4 models using equidistant quantile matching. Journal of Geophysical Research: Atmospheres, 115(D10): D10101. DOI:10.1029/2009JD012882

Maraun, D. et al., 2010. Precipitation downscaling under climate change: Recent developments to bridge the gap between dynamical models and the end user. Reviews of Geophysics, 48(3): RG3003. DOI:10.1029/2009RG000314

Mehrotra, R., Sharma, A., 2010. Development and Application of a Multisite Rainfall Stochastic Downscaling Framework for Climate Change Impact Assessment. Water Resources Research, 46(7): W07526. DOI:10.1029/2009WR008423

Mehrotra, R., Sharma, A., 2012. An improved standardization procedure to remove systematic low frequency variability biases in GCM simulations. Water Resources Research, 48(12): W12601. DOI:10.1029/2012WR012446

Mehrotra, R., Sharma, A., 2015. Correcting for systematic biases in multiple raw GCM variables across a range of timescales. Journal of Hydrology, 520(0): 214-223. DOI:http://dx.doi.org/10.1016/j.jhydrol.2014.11.037

Muerth, M.J. et al., 2013. On the need for bias correction in regional climate scenarios to assess climate change impacts on river runoff. Hydrol. Earth Syst. Sci., 17(3): 1189-1204. DOI:10.5194/hess-17-1189-2013

Ojha, R., Nagesh Kumar, D., Sharma, A., Mehrotra, R., 2013. Assessing Severe Drought and Wet Events over India in a Future Climate Using a Nested Bias-Correction Approach. Journal of Hydrologic Engineering, 18(7): 760-772. DOI:doi:10.1061/(ASCE)HE.1943-5584.0000585

Pegram, G., Bárdossy, A., 2013. Downscaling Regional Circulation Model rainfall to gauge sites using recorrelation and circulation pattern dependent quantile-quantile transforms for quantifying climate change. Journal of Hydrology, 504: 142-159. DOI:http://dx.doi.org/10.1016/j.jhydrol.2013.09.014

Prichard, D., Theiler, J., 1994. Generating surrogate data for time series with several simultaneously measured variables. Physical Review Letters, 73(7): 951-954. DOI:10.1103/PhysRevLett.73.951

Rajagopalan, B., Lall, U., 1999. A k-nearest-neighbor simulator for daily precipitation and other weather variables. Water Resources Research, 35(10): 3089-3101. DOI:10.1029/1999WR900028

Rocheta, E., Sugiyanto, M., Johnson, F., Evans, J., Sharma, A., 2014. How well do general circulation models represent low-frequency rainfall variability? Water Resources Research, 50(3): 2108-2123. DOI:10.1002/2012WR013085

Sharma, A., Mehrotra, R., Johnson, F., 2013. A New Framework for Modeling Future Hydrologic Extremes: Nested Bias Correction as a Precursor to Stochastic Rainfall Downscaling, Climate Change Modeling, Mitigation, and Adaptation. American Society of Civil Engineers, pp. 357-386. DOI:10.1061/9780784412718.ch13

Shumway, R.H., Stoffer, D.S., 2005. Time Series Analysis and Its Applications (Springer Texts in Statistics). Springer-Verlag New York, Inc.

Teutschbein, C., Seibert, J., 2012. Bias correction of regional climate model simulations for hydrological climate-change impact studies: Review and evaluation of different 
Table 1. RMSE for Autocorrelation Statistics ${ }^{*}$

\begin{tabular}{lcccc}
\hline \multicolumn{1}{c}{ Statistics } & Raw GCM & EQM & RNBC & FBC \\
& & & & \\
\hline Monthly Lag-1 Autocorrelation & 0.075 & 0.059 & $\mathbf{0 . 0 2 0}$ & 0.032 \\
Monthly Lag-2 Autocorrelation & 0.061 & 0.049 & 0.041 & $\mathbf{0 . 0 2 7}$ \\
Monthly Lag-3 Autocorrelation & 0.070 & 0.044 & 0.039 & $\mathbf{0 . 0 2 9}$ \\
Annual Lag-1 Autocorrelation & 0.139 & 0.142 & $\mathbf{0 . 0 9 3}$ & 0.115 \\
Annual Lag-2 Autocorrelation & 0.140 & 0.144 & 0.149 & $\mathbf{0 . 1 0 7}$ \\
Annual Lag-3 Autocorrelation & & & & \\
& 0.171 & 0.175 & 0.175 & $\mathbf{0 . 1 2 8}$ \\
\hline * The bias correction approach with the lowest RMSE across Australia is highlighted in bold & & \\
\hline
\end{tabular}




\section{Figure Captions}

Figure 1. Illustration of the FBC approach (a) two signals, $\mathrm{X}$ and $\mathrm{Y}$ plotted in the time domain; (b and c) autocorrelation plots of $\mathrm{Y}$ and $\mathrm{X}$; (d, e and $\mathrm{f}$ ), autocorrelation plots for the bias corrected time series obtained using $\mathrm{TBC} 1, \mathrm{TBC} 2$ and $\mathrm{FBC}$ approaches. The units and time scales for $\mathrm{X}$ and $\mathrm{Y}$ are arbitrary.

Figure 2. Scatter plots of mean (first row) and standard deviation denoted as SD (second row) at monthly, seasonal and annual time scales of raw GCM (first column), EQM (second column), RNBC (third column) and FBC (four column) bias corrected rainfall in the crossvalidation. Means and SD are divided by 100 for the presentation purpose. Plot with letter M denotes monthly, letter $\mathrm{S}$ is for seasonal and letter A is for annual statistics. The horizontal axis represents the observed, while the vertical axis represents the model simulated. Points on these plots denote each grid cell across Australia.

Figure 3. Boxplots of the RMSE for the autocorrelation function (ACF) based on the raw GCM, EQM, RNBC and FBC bias corrected monthly time series across Australia in the cross-validation. Outliers exceeding the length of the whiskers are shown as crosses.

Figure 4. The results for the APS metric from 24 to 60 months based on the raw GCM, EQM, RNBC and FBC bias corrected monthly time series across Australia in the cross validation. The bottom of each plot also shows the results for $\mathrm{APS}_{a b s}, \mathrm{APS}_{\text {pos }}$ and $\mathrm{APS}_{\text {neg }}$. Yellow to red areas indicate oversimulation of persistence while light blue to dark blue areas indicate undersimulation of persistence. Colour intensities reflect the magnitudes of oversimulation or undersimulation. Values in parentheses show the percentages of grid cells with the over- or under-simulation of rainfall persistence.

Figure 5. Three year and five year minimum total rainfalls of raw GCM (fist column) and bias corrected rainfall using EQM (second column), RNBC (third column), and FBC (four 
column) in the cross-validation.. Three year and five year minimum total rainfalls are rescaled by the annual observed standard deviations. First row shows 3-year minimum total rainfall, second row presents 5-year minimum total rainfall. The horizontal axis represents the observed, while the vertical axis represents the model simulated. Points on these plots denote each grid cell across Australia. The top left corner of each plot shows RMSE for the raw GCM and bias corrected results using EQM, RNBC and FBC across the grid cells. 

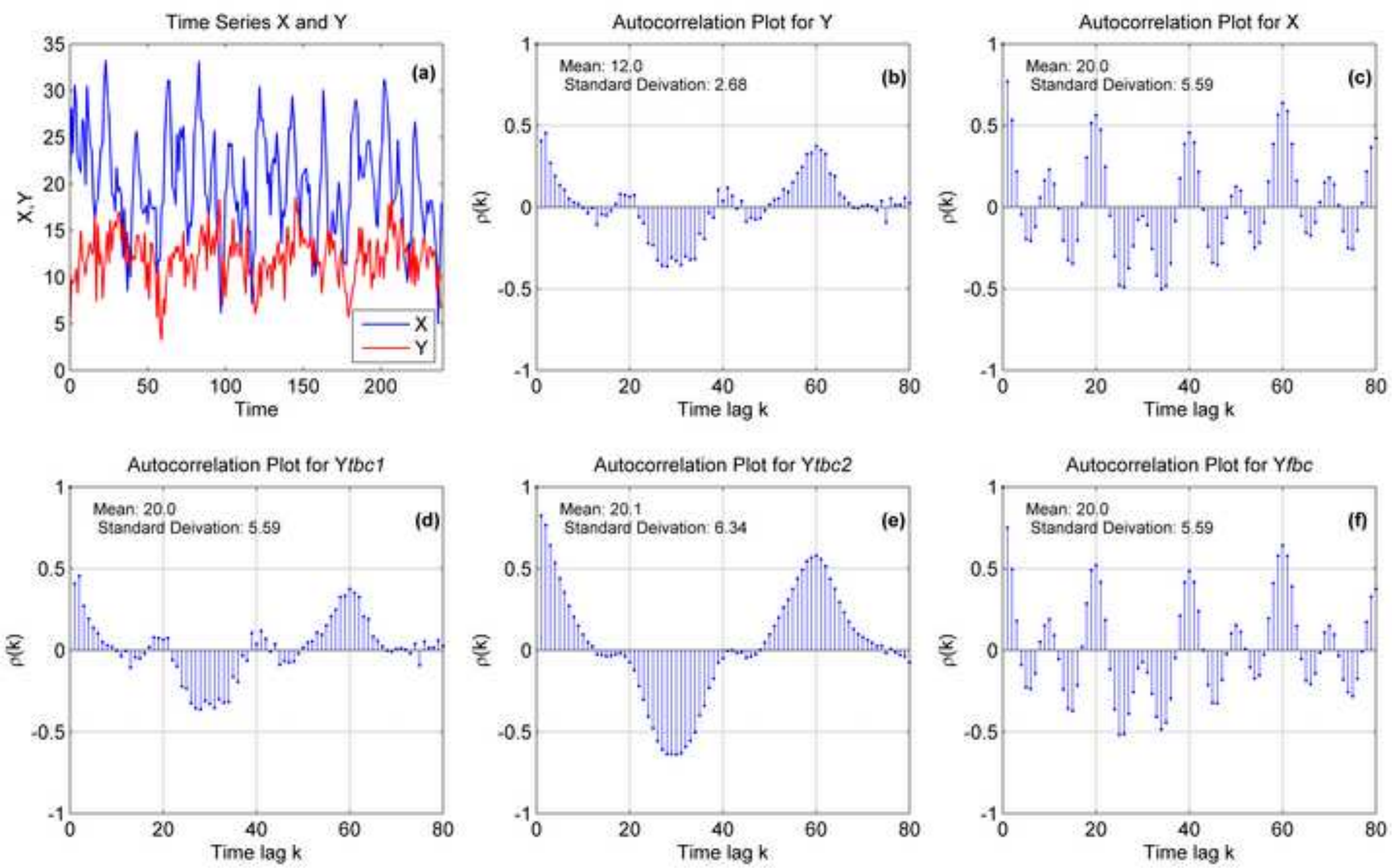

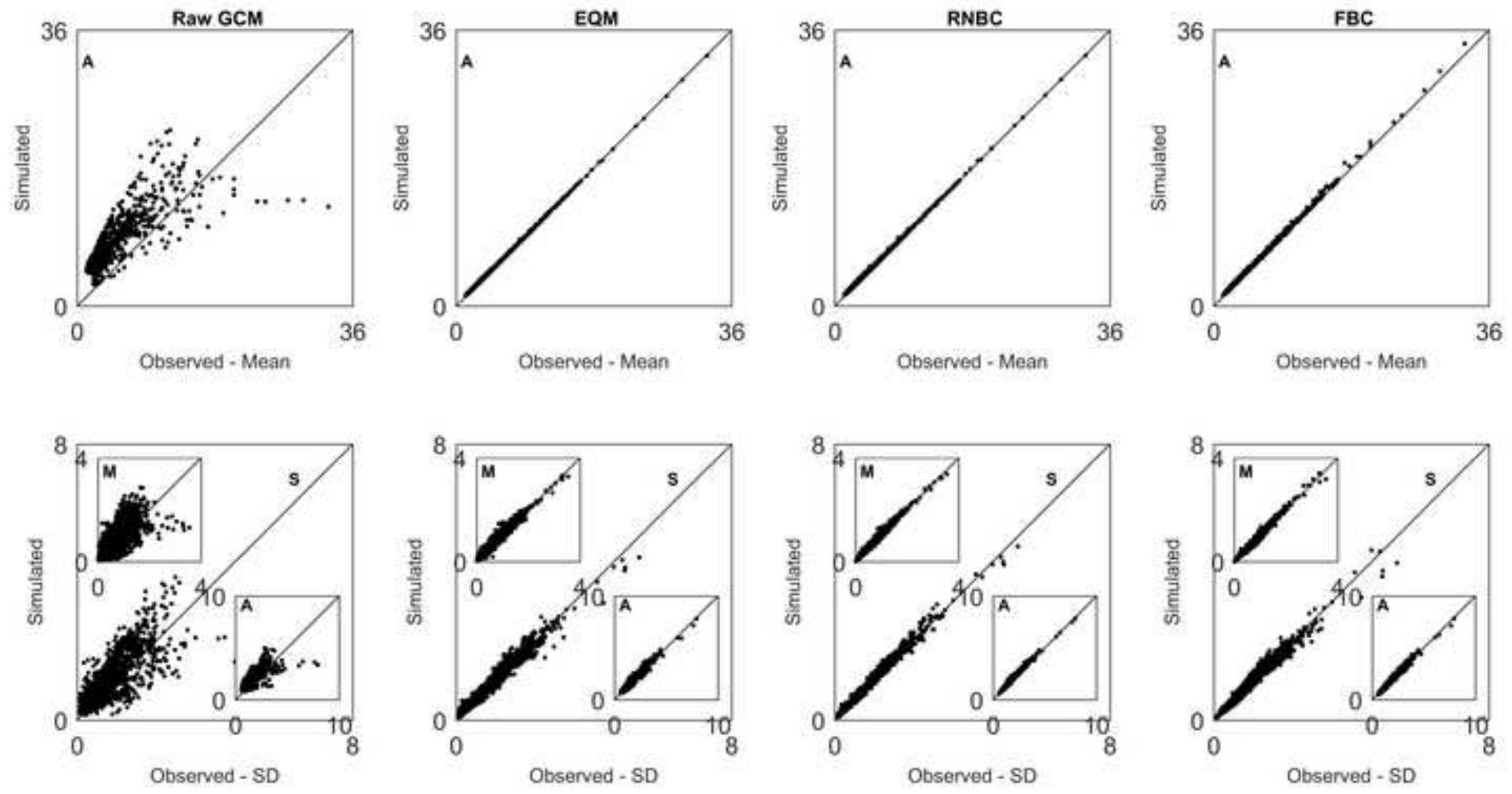


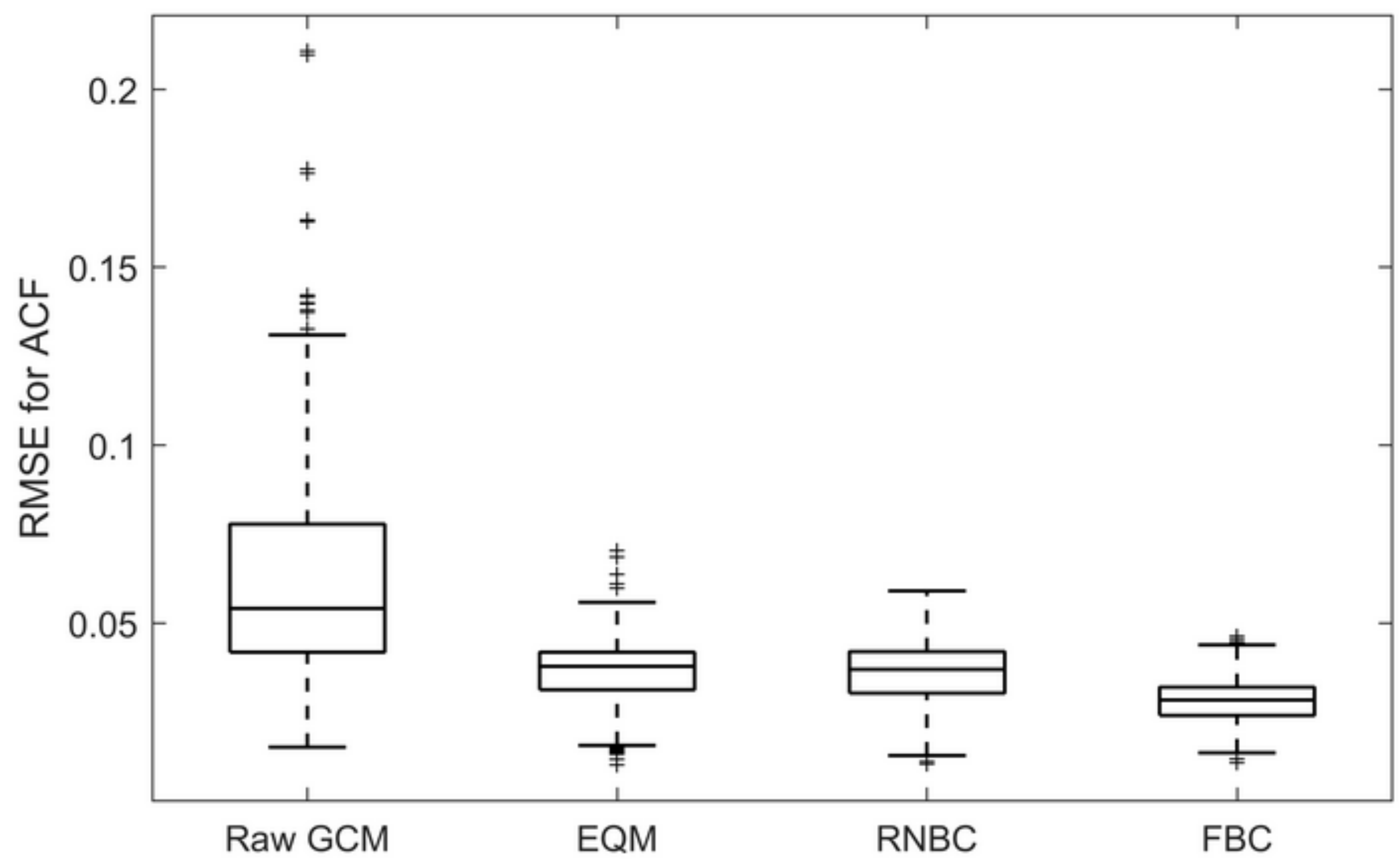



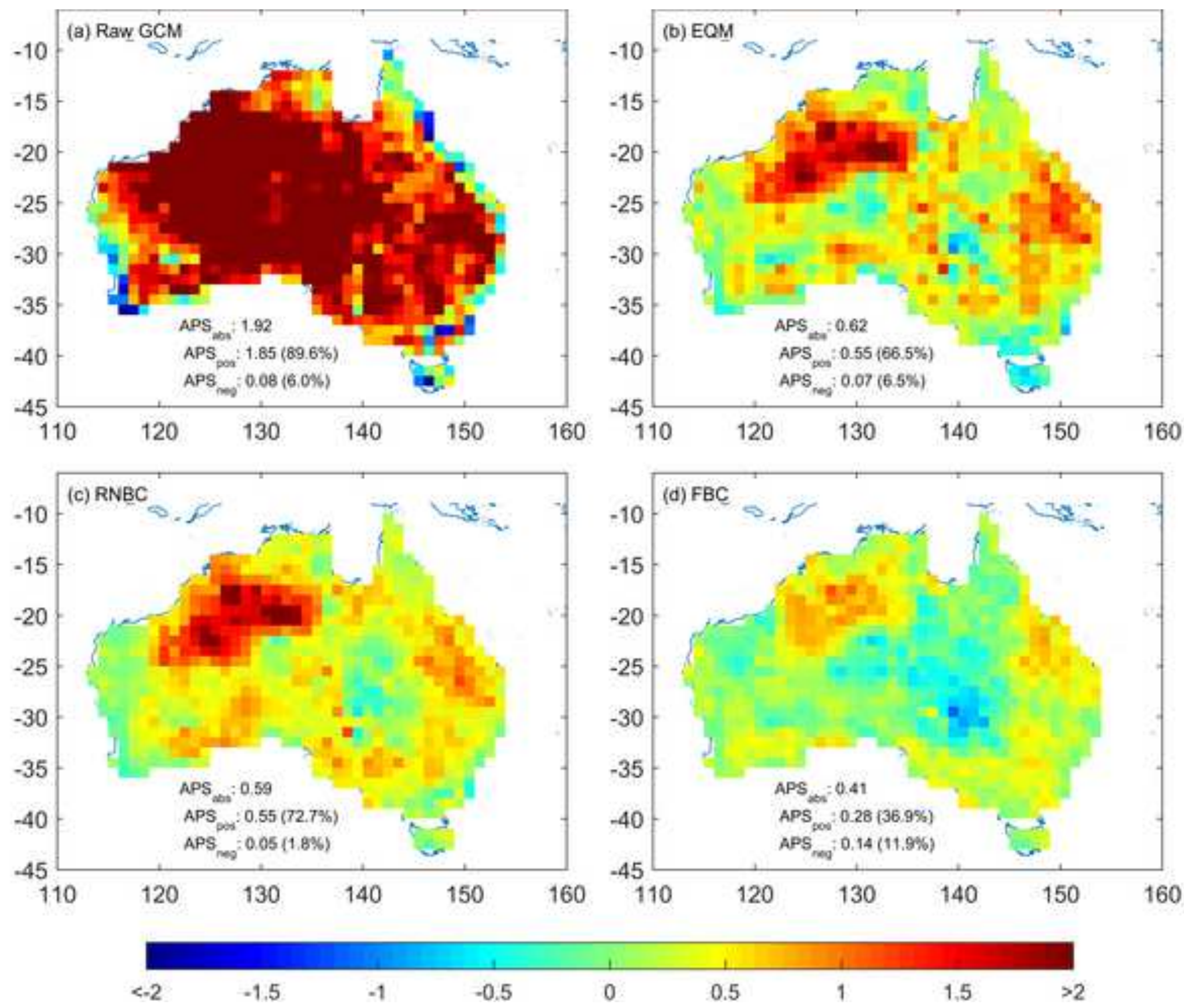

$-1$

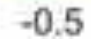

0

0.5

1,5 

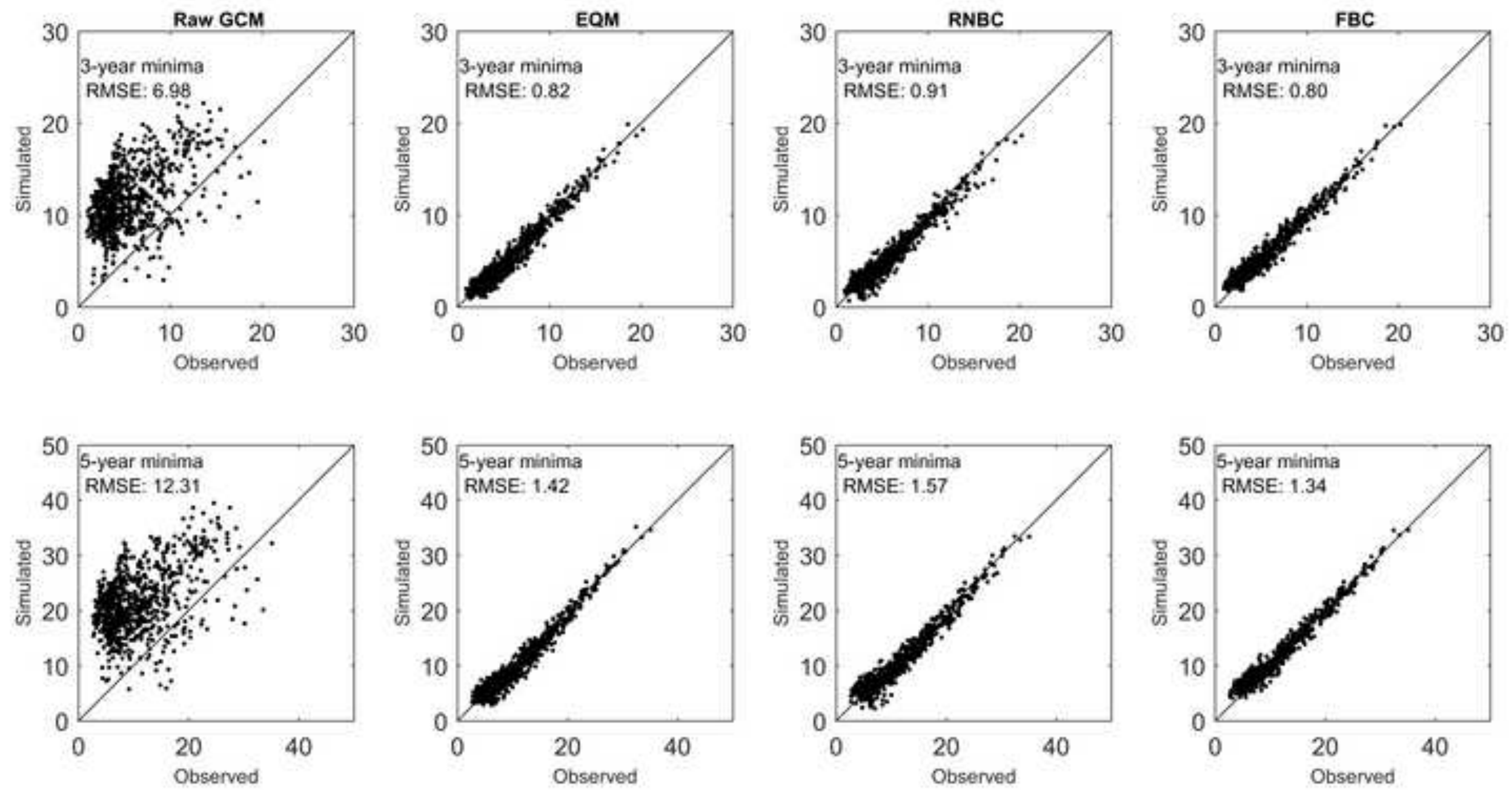